\title{
Sequential acid-catalyzed alkyl glycosylation and oligomerization of unprotected carbohydrates
}

Received 00th January 20xx, Accepted 00th January 20xx

DOI: $10.1039 / x 0 x \times 00000 x$

\begin{abstract}
Lea Spitzer, ${ }^{\mathrm{a}, \mathrm{b}}$ Sébastien Lecommandoux, ${ }^{\mathrm{b}}$ Henri Cramail, ${ }^{\mathrm{b}^{*}}$ and François Jérôme ${ }^{\mathrm{a}^{*}}$
An efficient method has been developed to synthesize end-functionalized oligosaccharides from unprotected monosaccharides in a one-pot/two-step approach. In the first step, mannose (and glucose) were functionalized with an alkyne group at the anomeric position through Fisher-glycosylation reaction with propargyl alcohol as glycosyl acceptor. In a second step, the functionalized monosaccharides were oligomerized and the experimental conditions optimized by varying the temperature, time and molar ratio between alcohol and sugar, to reach $\overline{D P_{n}}$ up to 8 . The obtained oligosaccharides showed complete propargylation at their reducing chain-end and were successfully coupled to oleic acid via Huisgen reaction, affording bio-based surfactants.
\end{abstract}

\section{Introduction}

Surfactants are ubiquitous molecules in our daily life with multiple applications in various sectors such as home and personal care, paint, food, medicine, water treatment, materials, etc. ${ }^{1-3}$ Due to the exponential increase of the world population, and also due to several emerging economies worldwide, our society's demand for surfactants is increasing dramatically. This market, of about 12 million tons per year, is expected to growth at an annual rate of $8.3 \% .{ }^{4}$ Because of environmental concerns, the manufacture of bio-based surfactants has become increasingly attractive, as it potentially provides more environmentally friendly products, although a full life cycle assessment should be systematically carried out to support this claim. ${ }^{3,}{ }^{5-9}$ The market of bio-based surfactants ${ }^{10}$ is about 3.5 million tons/year in Europe, a region where the annual growth rate of bio-based surfactants was $3 \%$ between 2008 and 2013, with alkylpolyglycosides (APG) registering the strongest growth. ${ }^{11-14}$

APG are industrially produced through the acid-catalyzed Fischer glycosylation reaction. ${ }^{14-17}$ This reaction couples unprotected monomeric sugars with long chain alkyl alcohols $\left(C_{5}-C_{18}\right)$, and releases a stoichiometric amount of water. During the Fisher glycosylation, the in situ produced monomeric alkyl glycosides tend to further react with unconverted sugars, leading to the formation of APG. Due to the release of water, concomitant oligomerization of the sugar head and hydrolysis of the glycosidic bond occur, leading to a thermodynamic equilibrium in which APGs are composed, on average, of 1.5 to 2.1 sugar units per fatty chain. Being able to increase the degree of polymerization $\left(\overline{D P_{n}}\right)$ of sugar units per fatty chain is of high interest, as it modifies the hydrophilic-

\footnotetext{
a. INCREASE/Institut de Chimie des Milieux et Matériaux de Poitiers, CNRS, Université de Poitiers, 1 rue Marcel Doré, 86073 Poitiers cedex 9, France. E-mail : francois.jerome@univ-poitiers.fr

b. INCREASE/Université de Bordeaux, CNRS, Bordeaux INP, LCPO, UMR 5629, F-

33600, Pessac, France.

+Electronic Supplementary Information (ESI) available: NMR, GC-FID, ESI-MS and MALDI-tof spectra. See DOI: 10.1039/x0xx00000x
}

lipophilic balance (HLB) of APG, and consequently their performances. For instance, this can tune their physicochemical properties in terms of water solubility, foaming properties, critical micelle concentrations, surface tensions etc. However, this elongation of the sugar moiety remains a very challenging task. The main hurdle is the in situ release of water which induces a rapid hydrolysis of glycosidic bonds, even when water is continuously distilled out. Furthermore, at the end of the reaction, removal of the excess of fatty alcohols by distillation is a very delicate step as it generally requires elevated temperature, leading to a partial degradation of APGs. So far, all attempts to produce APG with a $\overline{D P_{n}}$ larger than 2.1 failed. One should mention that enzymatic catalysis potentially paves the way to APGs with larger $\overline{D P_{n}}$, but the cost of the enzymes and the low reactor productivity hamper the implementation of these routes on a bigger scale. ${ }^{18,19,20,21}$

In this work, we propose an alternative straightforward strategy based on the use of propargyl alcohol (PGA), which serve not only as a glycosyl acceptor but also as a linker to subsequently introduce the fatty chain through a $100 \%$ atom economy copper-catalyzed Huisgen reaction. In contrast to fatty alcohols, at the end of the reaction, PGA can be conveniently separated by distillation at a low temperature limiting the degradation of APGs. Importantly, as sugars are more soluble in PGA than in fatty alcohols, oligomerization takes place in a larger extent during the evaporation step of PGA, leading to the formation of APG with a $\overline{D P_{n}}$ of 8 .

\section{Experimental}

\section{Chemicals}

D-(+)-Mannose (from wood, $\geq 99 \%$ ), D-(+)-Glucose ( $\geq 99.5 \%$ ) and Amberlyst-15 ( $\geq 90 \%, 0.355-1.18 \mathrm{~mm}, 1.7 \mathrm{ml} / \mathrm{L}$ capacity) were purchased from Sigma Aldrich, and dried under vacuum 
before use. GC-FID standards propargyl $\alpha$-D-mannopyranoside (PMan) and 1,6-Anhydro- $\beta$-D-mannopyranose (AMP) were purchased from Biosynth Carbosynth. Propargyl alcohol (99\%), BSTFA (+ $1 \%$ TMCS), D-Sorbitol ( $\geq 98 \%), 1,5,7-$ triazabicyclo[4.4.0]dec-5-ene (98\%, TBD), sodium azide (reagent plus $\geq 99.5 \%$ ), L-Ascorbic acid (99\%) and 3-bromo-1propanol (97\%) were purchased from Sigma Aldrich and used without further purification. Methyl oleate (MeOl $\geq 99.9 \%$ ) was obtained from Nuchekrep. Cuprisorb was purchased from Seachem and Copper(II) sulfate from ProLabo. Acetone, Acetonitrile, chloroform $\left(\mathrm{CDCl}_{3}\right)$, diethyl ether, dimethyl sulfoxide (DMSO), Ethyl acetate (EtOAc), petroleum ether (PE), pyridine and magnesium sulfate were purchased from Sigma Aldrich and used without further purification. Dialysis was operated using a Spectra/Por ${ }^{\circledR} 6$ MWCO 100-500 Da membrane (RC).

\section{Synthesis procedure}

General method of the Fischer glycosylation and oligomerization of mannose

\section{Step 1 -}

In a round-bottom flask, mannose $(5 \mathrm{~g}, 27.75 \mathrm{mmol})$ was dispersed in an excess of propargyl alcohol (5 eq.) and stirred at $80^{\circ} \mathrm{C}$ in the presence of amberlyst-15 $\left(4.2 \mathrm{~mol}^{\circ} \mathrm{H}^{+}\right)$for $3 \mathrm{~h}$. Then, the reaction was stopped and the as-obtained yellow/orange solution was separated by centrifugation from the catalyst. The as-recovered solution $(\mathrm{pH} 4)$ was used for the following step without further purification.

Step 2 -

Oligomerization was attained by removing the excess of propargyl alcohol through heating of the mixture of step 1 at $100{ }^{\circ} \mathrm{C}$ for $4 \mathrm{~h}$ under vacuum. Note that for analysis only, the trace amount of free propargyl alcohol was fully removed as follow: (PMan)n was dissolved in water and trace of propargyl alcohol was extracted with chloroform. The aqueous phase was then freeze-dried to give a brown-beige solid (4.3 g, 88\%). It could be additionally purified by filtration over active carbon, yielding a white powder (S1). $\oplus$ (SEC) $=2.03$; ATR-IR $=2116$ (C $\equiv C$ str.) (s); ${ }^{1} \mathrm{H}-\mathrm{NMR}\left(400 \mathrm{MHz}, \mathrm{D}_{2} \mathrm{O}\right): \delta=5.32-5.08(\mathrm{~m}, 1.66$ H), 5.08-5.01 $\left(d_{b}, 2 H\right), 4.97-4.90(d b, 3.23 H), 4.73(d, 0.44 H)$, 4.41-4.32 (m, 6.56 H, CH $)_{2}, 4.15-3.53(\mathrm{~m}, 49.86 \mathrm{H}, \alpha / \beta-\mathrm{H} 2$ $\alpha / \beta-H-6), 3.42-3.39$ (tq, $0.74 \mathrm{H}, \beta-\mathrm{H}-5), 2.95\left(\mathrm{t}_{\mathrm{b}}, 1 \mathrm{H}\right.$, alkyne); ${ }^{13} \mathrm{C}-\mathrm{NMR}\left(100.4 \mathrm{MHz}, \mathrm{D}_{2} \mathrm{O}\right): \delta=102.4,102.3,100.5,99.5,99.2$, 99.0, 98.6, 97.0, 78.7, 78.3, 78.1, 76.2, 73.2, 72.9, 72.7, 71.3, $70.6,70.5,70.3,69.9,69.8,66.7,66.4,65.4,60.9,54.7,54.6$.

\section{Characterization methods}

\section{Nuclear Magnetic Resonance (NMR)}

NMR spectra were measured on Bruker Advance III 400. Typically, 10 - $20 \mathrm{mg}$ of sample was diluted in $0.5 \mathrm{~mL}$ deuterated solvent before the measurement. Following reference values of the deuterated solvents were used: $D_{2} \mathrm{O}$ $\left({ }^{1} \mathrm{H}-\mathrm{NMR}: \delta=4.8 \mathrm{ppm}\right)$. The spectra data were analyzed using TopSpin (v. 4.0.9). Automatic phase correction and subsequently integration was applied.

\section{Matrix Assisted Laser Desorption/Ionisation-Time of flight (MALDI-tof)}

MALDI-MS spectra were performed by the CESAMO (Bordeaux, France) on an Autoflex maX TOF mass spectrometer (Bruker Daltonics, Bremen, Germany) equipped with a frequency tripled Nd:YAG laser emitting at $355 \mathrm{~nm}$. Spectra were recorded in the positive-ion linear mode and with an accelerating voltage of $19 \mathrm{kV}$. Samples were dissolved at $20 \mathrm{mg} / \mathrm{mL}$ (water: acetonitrile 70/30 + trifluoroacetic acid (TFA, 10 vol\%). The 2,5-dihydroxybenzoic acid (DHB) matrix solution was prepared by dissolving $10 \mathrm{mg}$ in $1 \mathrm{~mL}$ of acetonitrile. The solutions were combined in 10:1 v/v of matrix to sample. One to two microliters of the obtained solution were deposited onto the sample target and vacuumdried.

\section{Size Exclusion Chromatography (SEC)}

Polymer molar masses were determined by Size Exclusion Chromatography (SEC) using water as the eluent. Measurements in water were performed on an Ultimate 3000 system from Thermoscientific equipped with diode array detector DAD. The system also includes a multi-angle laser light scattering detector MALLS and differential refractive index detector $\mathrm{dRI}$ from Wyatt technology. Polymers were separated on two Shodex $\mathrm{OH}$ Pack $802.5(8 * 300)$ columns (exclusion limits from $500 \mathrm{Da}$ to $10000 \mathrm{Da}$ ) at a flowrate of 0.6 $\mathrm{mL} / \mathrm{min}$. Columns temperature was held at $25{ }^{\circ} \mathrm{C}$. Dextran from PSS was used as the standard.

\section{Attenuated Total Reflectance Infrared Spectroscopy (ATR-IR)}

Fourier transform infrared (FTIR) spectra were recorded on a Bruker VERTEX 70 instrument $\left(4 \mathrm{~cm}^{-1}\right.$ resolution, 64scans, DLaTGS MIR) equipped with a Pike GladiATR plate (diamond crystal) for attenuated total reflectance (ATR) at room temperature.

\section{Gas Chromatography with Flame-Ionization Detection (GC- FID)}

The composition of the mono- and disaccharide fraction was analyzed by GC-FID spectroscopy after the transformation of the sugar components into their corresponding per-Otrimethylsilyl (nonreducing sugars) or per-O-trimethylsilylated oxime (recuing sugars). The crude samples (in general 20-30 $\mathrm{mg}$ ) were dissolved in $1 \mathrm{~mL}$ of pyridine (containing $1 \mathrm{mg} / \mathrm{mL}$ sorbitol as internal standard). To $100 \mu \mathrm{L}$ of the resulting solution was then added $200 \mu \mathrm{L}$ of BSTFA ( $+1 \%$ TMCS) and the mixture was stirred at RT for $2 \mathrm{~h}$. During this operation, a white precipitate was observed, which was separated by filtration $(0,45 \mu \mathrm{m}$, regenerated cellulose) before injection into the apparatus. ${ }^{22}$ By this procedure, the non-reducing sugars give single peaks in the chromatogram, whereas reducing sugars afford two peaks for the corresponding syn and anti TMS-oximes. The identification and the quantification were achieved by comparison with authentic standards for which the response factors were obtained from the corresponding 
calibration curves using sorbitol as internal standard. The analysis was conducted with a GC Trace 1300 Gas Chromatograph (Thermoscientific) equipped with a split/splitness injector and flame ionization detector (FID). $\mathrm{A} \mathrm{H}_{2}$ flow rate of $34 \mathrm{~mL} / \mathrm{min}$ and an airflow rate at $350 \mathrm{~mL} / \mathrm{min}$ were used. The flow rate of carrier gas $\left(\mathrm{H}_{2}\right)$ was set at $1.2 \mathrm{~mL} / \mathrm{min}$. The temperature of the injection port and detector were set at $250{ }^{\circ} \mathrm{C}$ and $320^{\circ} \mathrm{C}$. The oven temperature was programmed to initiate at $90^{\circ} \mathrm{C}$ for $1 \mathrm{~min}$, then the temperature was raised to $220^{\circ} \mathrm{C}$ at a rate of $10{ }^{\circ} \mathrm{C} / \mathrm{min}$ and finally increased to $320^{\circ} \mathrm{C}$ at a rate of $40{ }^{\circ} \mathrm{C} / \mathrm{min}$ and held there for $5 \mathrm{~min}$. The injection volume was $1 \mu \mathrm{L}$ in the split injection mode (15:1). Separation was performed on a capillary column TRB-5MS (30 $\mathrm{m} \times 0.25 \mathrm{~mm} \times 0.25 \mathrm{~mm}$ film thickness) from Teknokroma with matrix 95\% Dimethyl-(5\%) diphenyl polysiloxane.

\section{Results and discussion}

The present study should be viewed as a two-step process in which step 1 is the acid-catalyzed glycosylation of monomeric sugars with PGA, and step 2 is the elongation of the sugar moiety (Scheme 1). As a case study, mannose was first selected. Transposition to glucose is discussed in a second part.

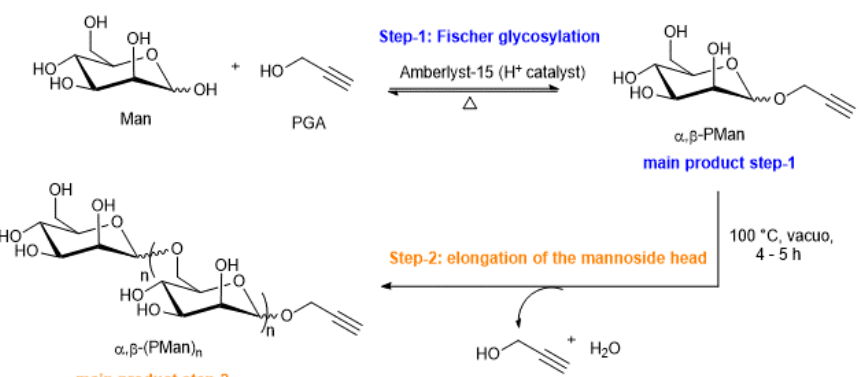

Scheme 1. Summarized method of the preparation of $(\mathrm{PMan})_{\mathrm{n}}$ through 2 steps: step $1=$ Mannose (1 eq.) + PGA (5 eq.) + Amberlyst-15 (4.2 mol\%), $80{ }^{\circ} \mathrm{C}, 1-12 \mathrm{~h}$; step $2=$ vacuum at $100{ }^{\circ} \mathrm{C}$ for $4-5 \mathrm{~h}$. For the sake of clarity, only the pyranoside form is represented.

\section{Step 1. Glycosylation of mannose with PGA}

In a first set of experiments, unprotected mannose was suspended in a 5-fold excess of PGA, this latter serving as both, glycosyl acceptor and solvent. The resulting mixture was stirred at $80{ }^{\circ} \mathrm{C}$ in the presence of Amberlyst-15, a solid acid catalyst $\left(4.2 \mathrm{~mol} \%\right.$ of $\left.\mathrm{H}^{+}\right)$. The reaction was monitored by gas chromatography (GC) over a period of $48 \mathrm{~h}$. Sequential oximation-trimethylsilylation derivatization procedure was used to calculate the conversion of mannose and to quantify the mono- and disaccharide fractions.

After $48 \mathrm{~h}$ (Figure 1), GC clearly revealed a conversion of mannose ( $\alpha$-mannose, peak $2, \beta$-mannose, peak 4 ) of $93 \%$, accompanied with the formation of $\alpha / \beta$ propargyl-(mono)mannopyranoside in 69\% yield (PMan, peak 3 and 5). Other detected products were disaccharides (peak 8), levomannosane (LVM, peak 1) and also minor products (such as furanosides peak $\mathbf{6}$ and acyclic acetals peak 7), in a relative proportion of $20 \%, 3 \%$ and $8 \%$, respectively. The retention times of standard chemicals are given in Figure S2 and Table S1. By means of size exclusion chromatography, propargyl(oligo)-mannosides (PMan) $n$ were also detected (Fig S3). In line with previous reports, the $\overline{D P_{n}}$ of (PMan) $)_{n}$, determined by ${ }^{1} \mathrm{H}$ NMR (see later for more information) was 1.3 , showing that oligomerization occurs in a very low extent under these conditions.

A plot of the PMan yields as a function of the reaction time is provided in Figure 2. It shows, that the PMan yield and the mannose conversion levelled off around $70 \%$ and $90 \%$, respectively, after $7 \mathrm{~h}$ of reaction. Extending the reaction time from 7 to $48 \mathrm{~h}$ did not result in a complete conversion of mannose, suggesting that the system has reached a thermodynamic equilibrium.
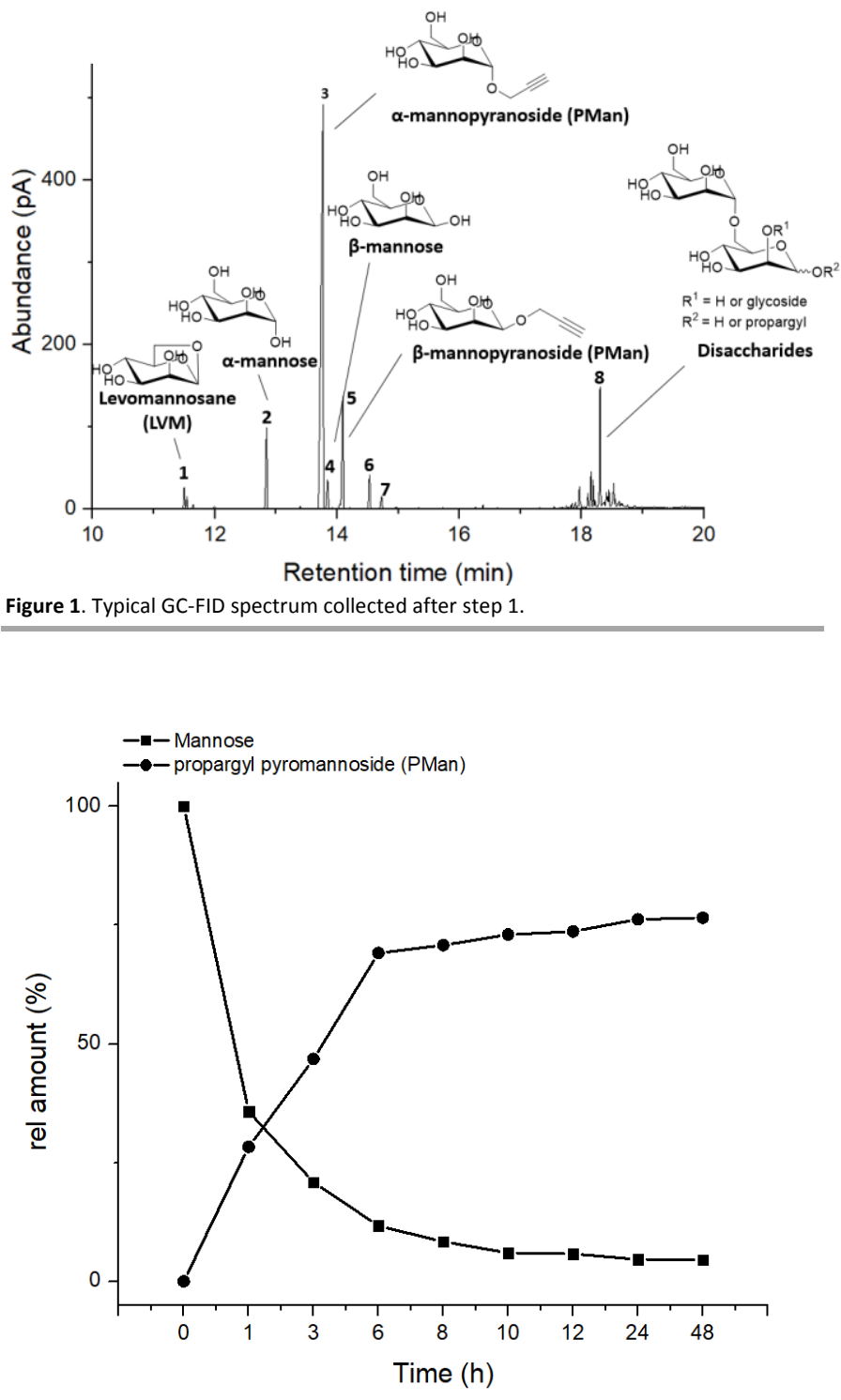

Figure 2. Mannose conversion and PMan yield as a function of the reaction time $\left(80^{\circ} \mathrm{C}\right.$, $\mathrm{PGA} /$ mannose molar ratio $=5$, Amberlyst-15 $\left(4.2 \mathrm{~mol}^{\mathrm{H}} \mathrm{H}^{+}\right)$. 
We next studied the influence of the temperature (Figure 3 ). Decreasing the reaction temperature from $100{ }^{\circ} \mathrm{C}$ to $80{ }^{\circ} \mathrm{C}$ obviously decreased the reaction rate (from $23 \mathrm{~mol} / \mathrm{h}$ to 9.5 $\mathrm{mol} / \mathrm{h}$ ). For instance, after $3 \mathrm{~h}$ of reaction, $90 \%$ and $79 \%$ of mannose were converted at $100{ }^{\circ} \mathrm{C}$ and $80^{\circ} \mathrm{C}$, respectively. When lowering the temperature further to $60{ }^{\circ} \mathrm{C}$, we faced problems of immiscibility between mannose and PGA.

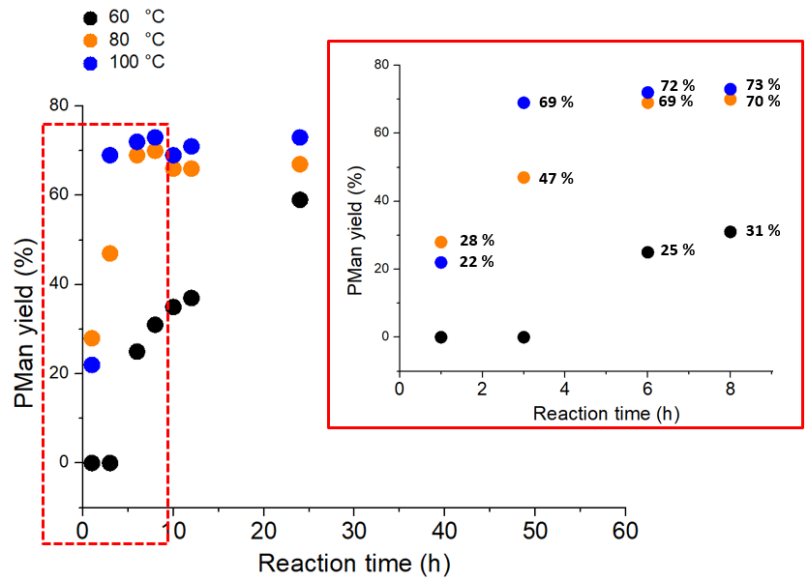

Figure 3. Effect of the reaction temperature on the PMan formation rate PGA/mannose molar ratio $=5$, Amberlyst- $15\left(4.2 \mathrm{~mol} \% \mathrm{H}^{+}\right)$.

However, at $60{ }^{\circ} \mathrm{C}$, the reaction started after $6 \mathrm{~h}$ and a conversion of mannose of about $80 \%$ was observed after $12 \mathrm{~h}$ of reaction $(2.5 \mathrm{~mol} / \mathrm{h})$. This $6 \mathrm{~h}$ induction period corresponds to the time to completely dissolve mannose in PGA at $60^{\circ} \mathrm{C}$.

Seeking for a compromise between the stability of mannose and the reaction rate, the reaction temperature was fixed at $80{ }^{\circ} \mathrm{C}$ in the following experiments. Note that decreasing the $\mathrm{PGA} /$ mannose molar ratio from 5 to 3 slowed down the reaction rate from $6.2 \mathrm{~mol} / \mathrm{h}$ to $4.5 \mathrm{~mol} / \mathrm{h}$, tentatively attributed to an increase of the reaction media viscosity. However, it did not significantly impact either the PMan yield (Figure 4 ) or the $\overline{D P_{n}}$ of (PMan) $n$ recovered in step 1 , confirming that in situ released water prevents oligomerization in a large extent.

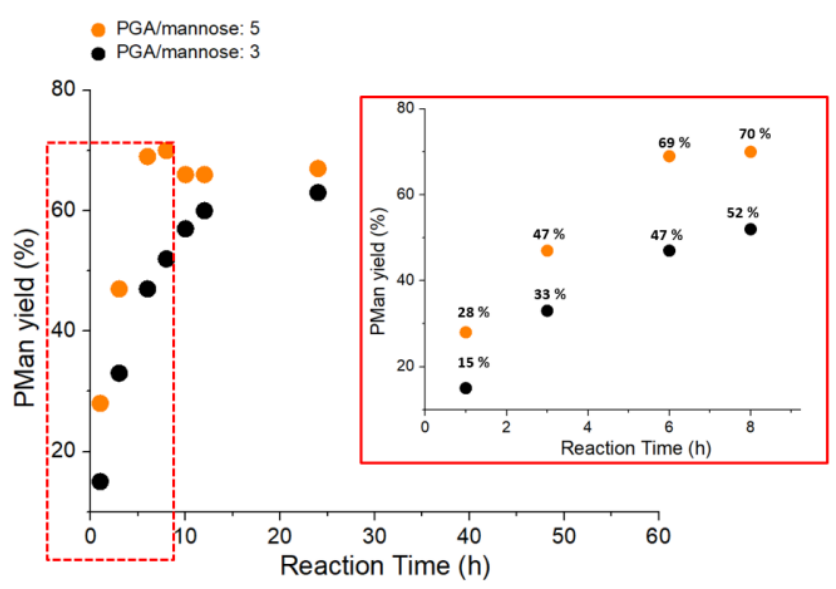

Figure 4. Effect of the $\mathrm{PGA} /$ mannose molar ratio on the PMan formation rate $80{ }^{\circ} \mathrm{C}$ Amberlyst-15 $\left(4.2 \mathrm{~mol} \% \mathrm{H}^{+}\right)$.

\section{Step 2. Oligomerization of the sugar head}

In order to increase the $\overline{D P_{n}}$ of PMan, the reaction mixture was then heated under vacuum at $100{ }^{\circ} \mathrm{C}$ to distillate out the excess of PGA and the in situ released water. The time needed to evaporate the excess of PGA ( $4 \mathrm{~h}$ ) restricted the duration of the reaction. Effect of this treatment on the average $\overline{D P_{n}}$ was monitored by SEC. As illustrated on Figure 5, after $4 \mathrm{~h}$ under vacuum, a shift towards high molar masses was clearly observed, a result consistent with an elongation of the sugar moiety. This treatment was accompanied by the formation of a beige-brownish powder. After complete removal of PGA, only $5-8 \%$ of monomeric species remained (determined by gas chromatography). The molar masses of the total batch was $1353 \mathrm{~g} / \mathrm{mol}$ (i.e. $\overline{D P_{n}}=8$ ) with a dispersity, $\Theta$, around 2 .

Note that distilled PGA can be reused for further glycosylation reactions in step 1 , but only after drying over magnesium sulfate to remove trace of water.

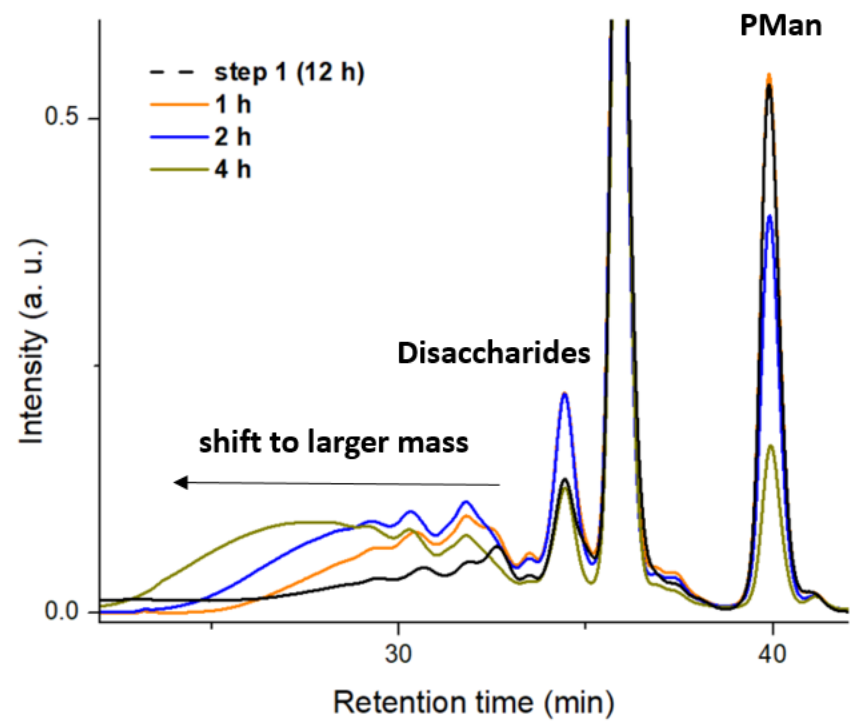

Figure 5. Overlaid SEC (measured against dextran-standards in $\mathrm{H}_{2} \mathrm{O}$ at $25^{\circ} \mathrm{C}$ ) spectra during step $2\left(100^{\circ} \mathrm{C}\right.$, vacuum).

To get more information on the structures of oligomers formed, NMR investigations were performed (Figure 6). Owing to the low field shift of the alkyne proton (2.95 ppm), the endgroup method could be employed to calculate the degree of oligomerization, and indirectly the average molar mass. The $\overline{D P_{n}}$ and $\overline{M_{n}}$ values were calculated from the ${ }^{1} \mathrm{H}-\mathrm{NMR}$ spectra using the following equations:

$$
\begin{gathered}
\overline{D P_{n}}=\frac{(\mathrm{H} 2+\mathrm{H} 3+\mathrm{H} 4+\mathrm{H} 5+\mathrm{H} 6)}{6} \\
\overline{M_{n}}=179+M_{0} D P_{n}+201
\end{gathered}
$$

where $M_{0}$ is the repeating unit with a molar mass of 162 $\mathrm{g} / \mathrm{mol}$. The values of $179 \mathrm{~g} / \mathrm{mol}$ and $201 \mathrm{~g} / \mathrm{mol}$ derive from the end groups of (PMan) ${ }_{n}$ (Fig. S4). A detailed analysis by one and two-dimensional NMR spectroscopy, including the ${ }^{n} J_{H-H}$-and 
${ }^{\mathrm{n}} J_{\mathrm{C}-\mathrm{H}}$ couplings, is given in the supplementary information (Fig.

S5 - S7).

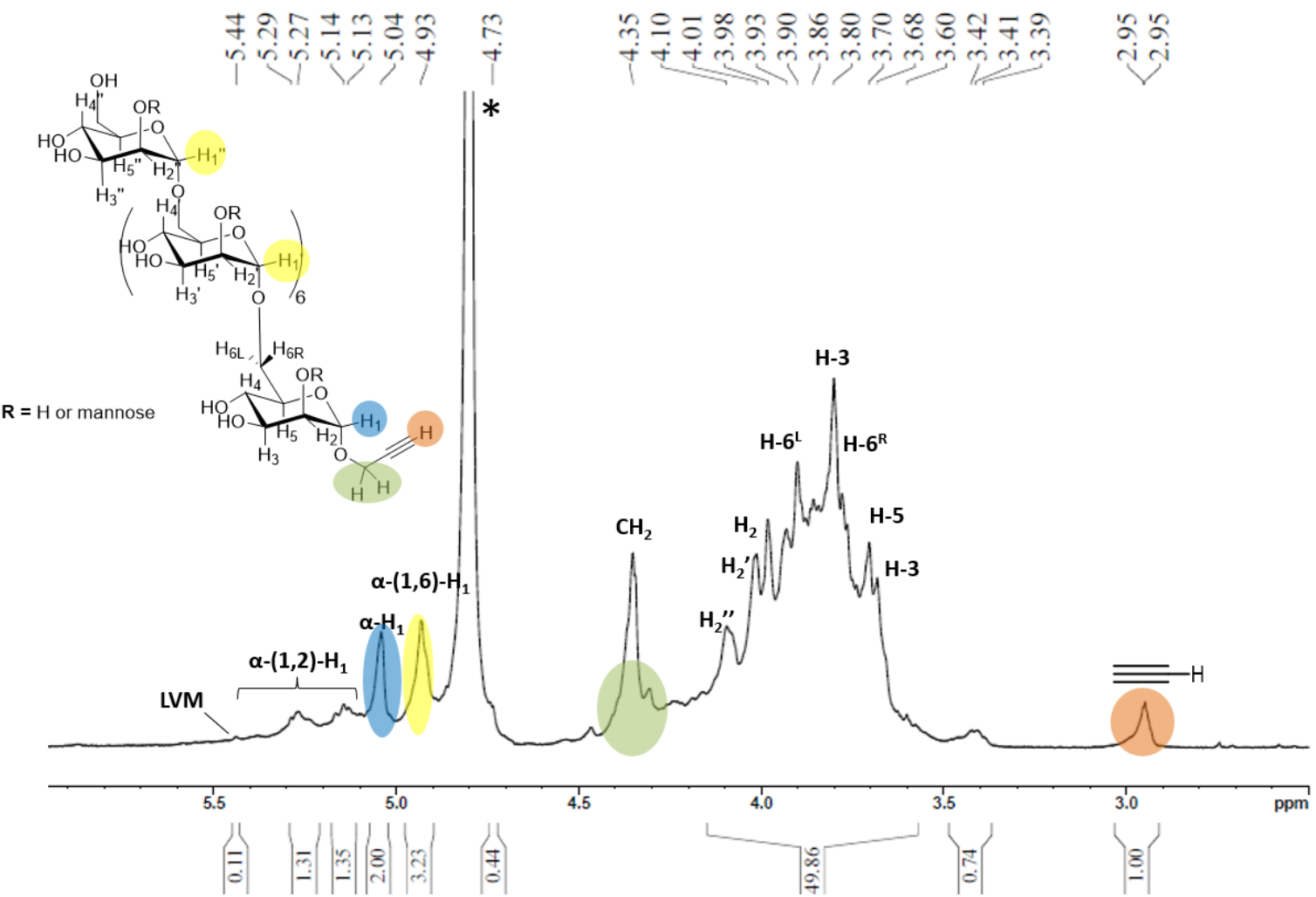

Figure 6. ${ }^{1} \mathrm{H}-\mathrm{NMR}\left(400 \mathrm{MHz}, \mathrm{D}_{2} \mathrm{O}\right)$ spectrum of $(\mathrm{PMan})_{n} ; \overline{D P_{n}}=8, \overline{M_{n}}=1353 \mathrm{~g} / \mathrm{mol}$.

The signals at $2.95 \mathrm{ppm}$ (brown, $\mathrm{HC} \equiv$ ) and $4.32-4.41 \mathrm{ppm}$ (green, $\mathrm{CH}_{2}$ ) confirmed the coupling of oligomannosides with PGA (Figure 6). Indeed, these signals chemically shifted downfield as compared to pure PGA (2.80 ppm ( $\mathrm{HC} \equiv$ ) and $\left.4.20 \mathrm{ppm}\left(\mathrm{CH}_{2}\right)\right)$. The broad peak at $5.01-5.08 \mathrm{ppm}$ was assigned to the anomeric proton (blue, $\alpha-\mathrm{H}-1$ ) located in $\alpha$ position at the chain end of (PMan $)_{n}$. Assignment of this proton could be further confirmed either with the reference signal of pure monomeric PMan at 5.06 - 5.05 ppm (Fig. S8) ${ }^{23 \text {, }}$ ${ }^{24}$ or with a coupling constant of $4 \mathrm{~Hz}$ between $\alpha-\mathrm{H}-1$ and the $\mathrm{CH}_{2^{-}}$of PGA in the NMR HMBC spectrum (Fig. S7). The spectrum in Figure 6 showed additional peaks in the range of 4.90 to $5.32 \mathrm{ppm}$. This region is characteristic for the anomeric protons and were thus attributed to the glycosidic linkages between the anhydromannose units in the oligomannoside chain. The most intense peak at $4.93 \mathrm{ppm}$ (yellow on figure 6) was assigned to the anomeric proton of a $\alpha-(1,6)$-glycosidic linkage. This claim is supported by NMR NOESY experiments (Fig. S6) showing a proton-proton long range coupling of this anomeric proton with the $\mathrm{H}-6$ protons of the anhydromanose unit. The $\alpha$ configuration was also supported by the HMBC spectrum with a carbon-proton coupling of $171.32 \mathrm{~Hz}$ (Fig.
S7). ${ }^{25}$ The additional multiplet signals appearing between 5.08 - $5.32 \mathrm{ppm}$ were tentatively attributed to the $\alpha$-(1,2)-glycosidic linkages, as COSY- and NOESY NMR spectroscopy (Fig. S5, S6) showed an exclusive $\mathrm{H}, \mathrm{H}$-coupling with $\mathrm{H}-2$ protons of the mannose ring (4.1 - $3.98 \mathrm{ppm})$. On the basis of signal intensity, the oligomannoside chain is dominantly assembled through $\alpha$ $(1,6)$-glycosidic bonds and, in a lower extent, through $\alpha-(1,2)$ glycosidic linkages (Fig. S9, Table S2). One should note that the additional peak at $4.73 \mathrm{ppm}$ suggests the existence of $\beta$ glycosidic linkage. This was supported by the proton-proton long range coupling to $\beta-\mathrm{H}-5$ (3.41 ppm) in the NMR NOESY spectrum (Fig. S6). However, due to the poor intensity in the 2D-NMR spectra, they were accounted to very low percentages. Integration of the different anomeric signals in ${ }^{1} \mathrm{H}$ NMR spectrum (Figure 6 ) suggested that the oligomannoside chain is composed of $60 \%$ of $\alpha$ - $(1,6)$-linkages, $24 \%$ of $\alpha-(1,2)$ linkages, whereas the $\beta-(1,6)$ and $\beta-(1,2)$ linkages representing only $8 \%$. Another small doublet found at $5.44 \mathrm{ppm}$ accounted to $5 \%$ relative to all anomeric protons and was assigned to the anomeric proton of levomannosane (LVM). This chemical composition is also supported by ${ }^{13} \mathrm{C}$ NMR (Fig. S10). This structure is actually very similar to what was previously
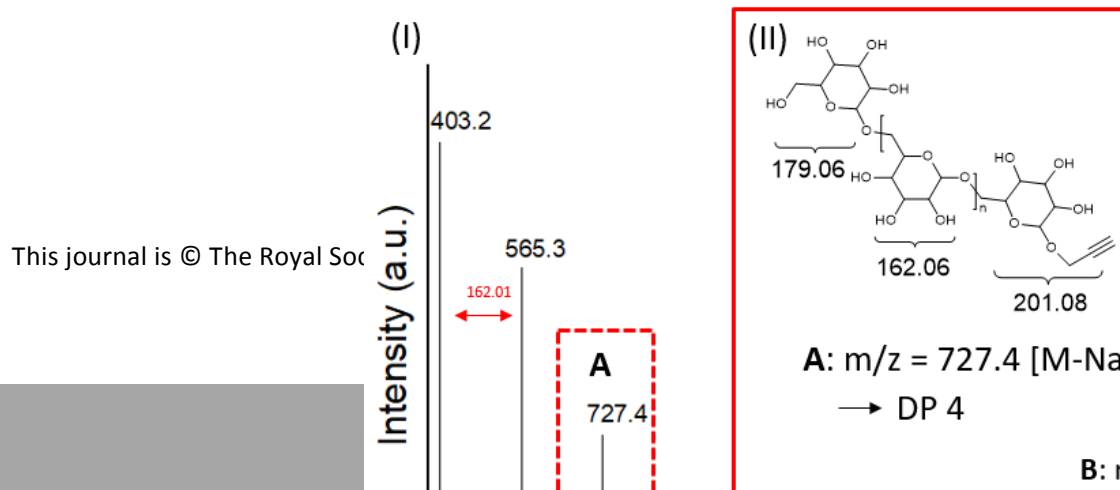

A: $m / z=727.4[\mathrm{M}-\mathrm{Na}]^{+}$ 
Figure 7.: (I) MALDI-TOF spectrum of (PMan) ${ }_{n}$ recovered after step 2.

observed in the oligomerization of mannose by high frequency ultrasound $^{26}$, the 1-6 linkages being thermodynamically the most favorable ones.

To further support this chemical composition, samples were analyzed by MALDI-TOF spectrometry (Figure 7). Data showed a series of monoanionized peaks with sequential increments of $\mathrm{m} / \mathrm{z}=162 \mathrm{Da}$ (corresponding to anhydromannose residue as repeating unit). This series was assigned to the (PMan $)_{n}$ and DP up to 12 were detected, which corresponds to a $\overline{D P_{n}}$ of 8 as calculated by NMR (equation (1)). Within the noise of the MS spectra, a second series, with $\mathrm{m} / \mathrm{z}$ lower than 56 as compared to (PMan) $)_{n}$ was observed and corresponded to the formation of an oligomannoside terminated by a dehydrated anydromannose residue. The formation of this oligomannoside series remained however quasi negligible, as also supported by NMR investigations. This combined NMR-mass analysis led us to conclude that the as-obtained oligomannosides are (1) fully propargylated at their terminal end, (2) mainly linked through $\alpha-(1,6)$-linkages and (3) exhibit an $\overline{D P_{n}}$ up to 8 . By means of DSC analysis, the $\mathrm{Tg}$ of (PMan) 8 was found to be $120^{\circ} \mathrm{C}$.

To further increase the $\overline{D P_{n}}$ of (PMan) $)_{n}$, step 1 was stopped before complete conversion of mannose; this should facilitate the reaction of PMan with unreacted mannose in step 2. When the glycosylation of mannose with PGA was stopped after 1 3 h at $80{ }^{\circ} \mathrm{C}$ in step $1,21-36 \%$ of free mannose remained unreacted. When vacuum (step 2) was applied at that stage, the unreacted free mannose randomly polymerized either with PMan or other molecule of mannose. Unfortunately, a mixture of $(\text { PMan })_{n}(76 \%)$ and terminal free oligomannoside (12\%) along with $12 \%$ of LVM were obtained. To ensure elongation of the sugar moiety and full glycosylation with PGA at the terminal position, it is thus mandatory to achieve a nearly complete conversion of mannose in step 1 prior to step 2, suggesting also that step 1 is reversible under vacuum (see later for more information). Best conditions in step 1 are $80^{\circ} \mathrm{C}$, a PGA/mannose molar ratio of 5 and $3 \mathrm{~h}$ of reaction, leading to the formation of PMan with 65\% yield (step 1) and (PMan) with a $\overline{D P_{n}}$ of 8 in step 2 (Figure 6).

\section{Plausible reaction mechanism}

On the basis of all these results, we suggest a plausible reaction mechanism (Scheme 2), inspired by the most accepted proposals of glycosylation in the literature. ${ }^{27,} 28,29$ In step 1 , mannose is protonated by amberlyst-15 to form the oxocarbenium ion, which is then rapidly trapped by PGA to form PMan. The elongation of the mannose moiety to form APG occurs in a very low extent in step 1, mainly due to the excess of PGA and the in situ release of water which prevents oligomerization reaction. In step 2, PMan is reprotonated, partly regenerating the oxocarbenium ion, as previously suggested by Mamidyala et al., The Journal of organic chemistry, 2009, 74, 8417-8420. et al..$^{30}$ During the distillation of PGA (and water) under vacuum, the reaction of the oxocarbenium ion with PMan is statistically more likely to occur, leading to the formation of (PMan) $)_{n}$ with an $\overline{D P_{n}}$ of 8 . To confirm the mechanism of step 2, PMan was prepared, isolated by chromatography over silica gel (acetonitrile/water $(10-30$ vol\%)) and freeze dried for at least two days. Then, purified PMan was dissolved in PGA (5 eq.) and stirred at $80^{\circ} \mathrm{C}$ for $1 \mathrm{~h}$ (step 1 ) with Amberlyst-15 prior to heating the mixture at $100{ }^{\circ} \mathrm{C}$ under vacuum for $4 \mathrm{~h}$ (step 2). Under these conditions, PMan was converted to (PMan) $\mathrm{n}$ with a $\overline{D P_{n}}$ of 4 , in $85 \%$ yield (Fig. S11). These findings support that PMan could be depropargylated to form the oxocarbenium ion back, which can further react with another species of PMan, resulting in an elongation of the mannoside chain. One should note that this oligomerization reaction is possible thanks to the solubility of mannose and APG in PGA. In contrast, with fatty alcohols, this 
elongation was not observed in step 2 due to solubility issue, i.e. precipitation (and/or degradation) of monoalkylglycosides and sugars upon evaporation. The proposed reaction mechanism also explains the formation of LVM as a minor product, resulting from the internal cyclization of the oxocarbenium ion.

Note that Amberlyst-15 can be replaced by strongly acidic $\mathrm{H}_{2} \mathrm{SO}_{4}$ or Aquivion PFSA. However, due to the high acid strength of these acids, the proton loading has to be reduced from $4.2 \mathrm{~mol} \%$ to $0.13 \mathrm{~mol} \%$ to avoid fast and uncontrolled degradation of sugars, in particular in step 2.

\section{Transposition to glucose}

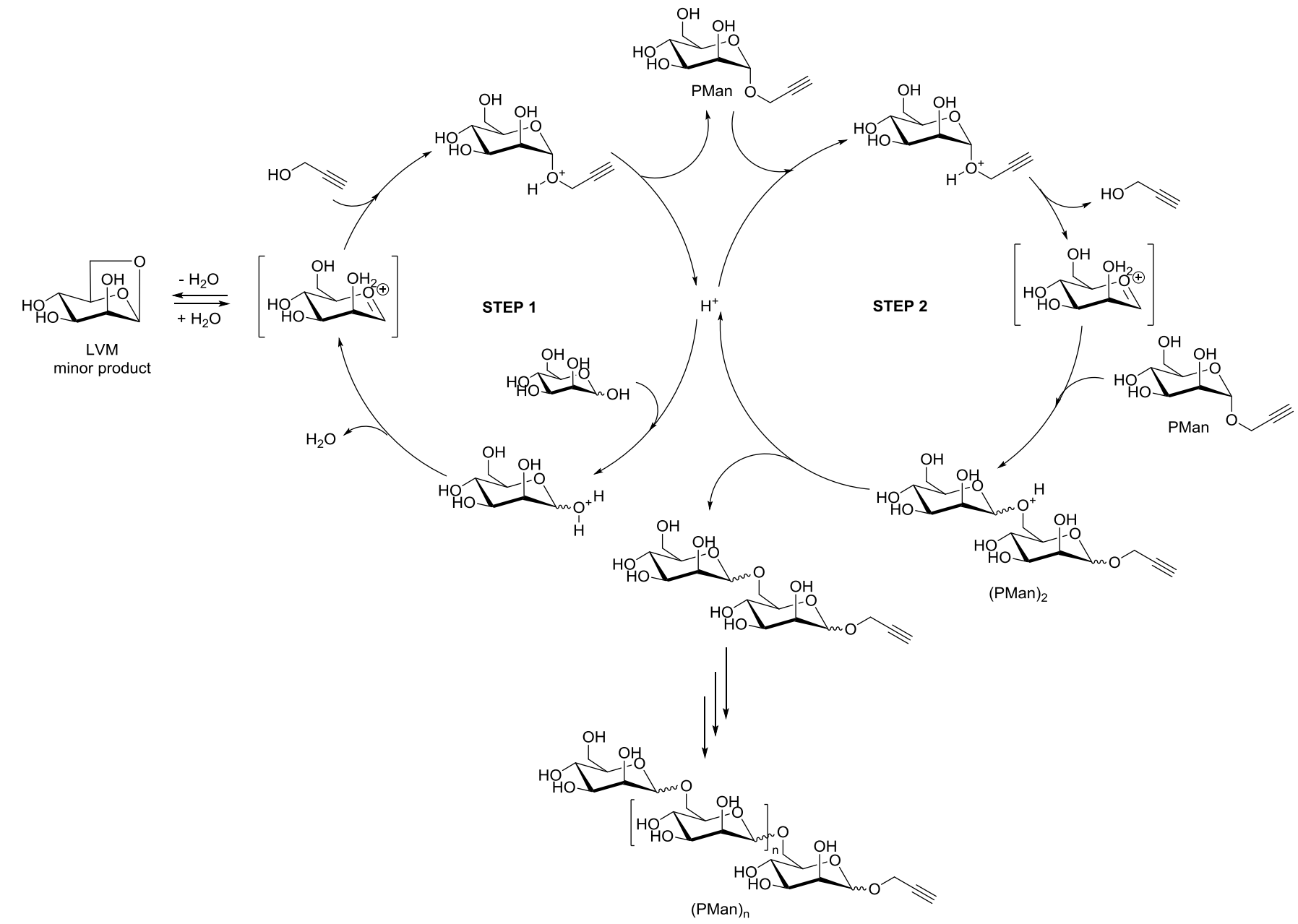

Scheme

2.

Proposed

reaction

mechanism

for

step

and

step

As stated in the introduction section, the alkyne group at the chain-end of the prepared oligosaccharides (mannose and glucose) enables a direct access to amphiphiles. To this purpose, the well-known 'azide-alkyne Huisgen cyclo-addition' presents an attractive method due to its atom-economy and quantitative conversions. ${ }^{31}$ As demonstrated in previous studies $^{5,32}$, this method is frequently utilized to prepare amphiphilic block copolymers and opens up further applications of the as-prepared oligosaccharides. We show in a proof-of-concept experiment (Scheme 3) the successful addition of a long chain fatty acid (oleic acid) via click chemistry to the mannose-oligomers (for more details, see Fig. S14 - S18). The structure of the as-obtained amphiphile was evidenced by ${ }^{1} \mathrm{H}-\mathrm{NMR}$ (Fig. S18) with the appearance of the characteristic triazole peak at $8.1 \mathrm{ppm}$ and the analysis by MALDI-tof, which confirmed no depolymerization during the 
click reaction (Fig. S19). This presents a powerful strategy to prepare amphiphilic APGs while avoiding the in situ depolymerization of oligosaccharides, as commonly observed with other functionalization methodologies.

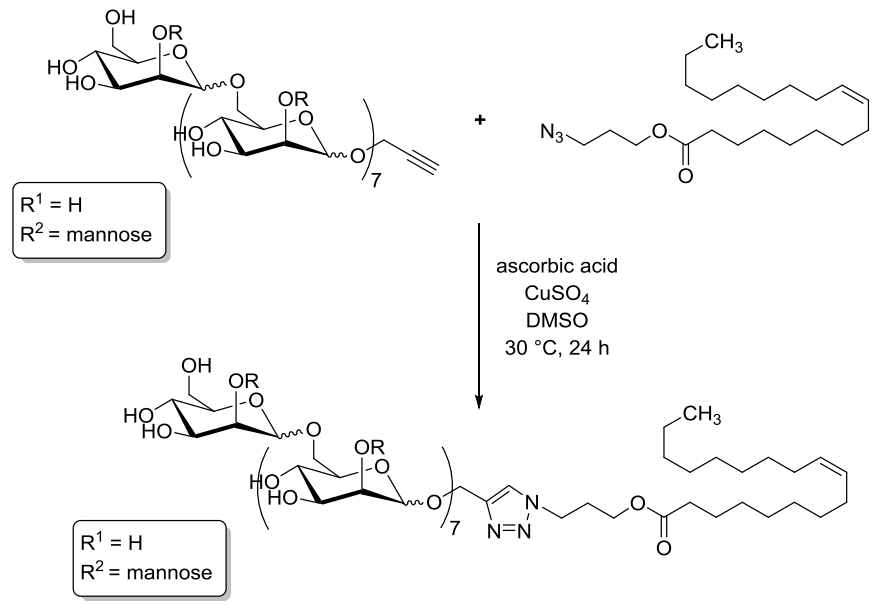

propargyl-(oligo)-mannoside-b-oleic acid

Scheme 3. Preparation of amphiphiles by the copper-catalyzed addition of oleic acid to $(\text { PMan })_{n}$ via click-chemistry.

\section{Conclusions}

In this work, we demonstrated that the acid catalyzed (amberlyst-15) glycosylation reaction of unprotected monosaccharides (mannose, glucose) and propargyl alcohol, afforded fully functionalized propargylated glycosides with $\overline{D P_{n}}$ up to 8 in $88 \%$ yield. The fine control of temperature, time and molar ratio of sugar to alcohol, could tune the $\overline{D P_{n}}$. Whereas the preparation of APGs with a $\overline{D P_{n}}$ of more than 2 remains to date a huge challenge ${ }^{17,27-29}$, the proposed method could expand the molar mass and maintain complete glycosylation of the sugars at the same time. The distillation of water along with propargyl alcohol in step 2, resulted to be the key-step for the oligomerization. The elucidation of the structure by NMR spectroscopy, strongly suggests that the mannose-oligomers are mainly linked through $\alpha-(1,6)$ glycosidic bonds, along with branching points at $\mathrm{OH}-2$.

In a 'proof-of-concept' experiment, the alkyne function at the terminal end of the oligomers was exploited to add a longchain fatty acid via Huisgen catalyzed 'Click-Chemistry' to the sugars. We presented thereby an alternative method to synthesize oligosaccharide-based amphiphiles, that could be employed as bio-based surfactants. Further developments with respect to the synthesis of various oligosaccharide-based amphiphiles and investigations of their properties will be discussed in forthcoming contributions.

\section{Conflicts of interest}

There are no conflicts to declare.

\section{Acknowledgements}

The authors thank the CNRS, the Région Nouvelle Aquitaine, the University of Poitiers and the University of Bordeaux for their financial supports. Authors are also grateful to the FR CNRS INCREASE for funding. CESAMO, Institut des Sciences Moléculaires (University of Bordeaux, Talence (33), France) are also acknowledged for MALDI-TOF MS experiments.

\section{Notes and references}

1. D. G. Hayes, D. K. Solaiman and R. D. Ashby, Biobased Surfactants: Synthesis, Properties, and Applications, Elsevier, 2019.

C. Stubenrauch, Current Opinion in Colloid \& Interface Science, 2001, 6 $160-170$. M. Henkel and R. Hausmann, in Biobased Surfactants (Second Edition), eds. D. G. Hayes, D. K. Y. Solaiman and R. D. Ashby, AOCS Press, 2019, DOI: https://doi.org/10.1016/B978-0-12-812705-6.00002-2.

N. Albany, Transparency Market Research, 2012, 82.

C. Schatz and S. Lecommandoux, Macromol Rapid Commun, 2010, 31 1664-1684.

S. de Medeiros Modolon, I. Otsuka, S. b. Fort, E. Minatti, R. Borsali and S. Halila, Biomacromolecules, 2012, 13, 1129-1135.

I. Otsuka, K. Fuchise, S. Halila, S. Fort, K. Aissou, I. Pignot-Paintrand, Y Chen, A. Narumi, T. Kakuchi and R. Borsali, Langmuir, 2010, 26, 2325-2332. J. Scheibel, 2007

C. Schatz, S. Louguet, J. F. Le Meins and S. Lecommandoux, Angewandte Chemie International Edition, 2009, 48, 2572-2575.

https://www.gminsights.com/industry-analysis/biosurfactants-marketreport).

J. Schulte, S. Enders and K. Quitzsch, Colloid and Polymer Science, 1999, 277, 827-836.

12. E.-M. Kutschmann, G. Findenegg, D. Nickel and W. Von Rybinski, Colloid and Polymer Science, 1995, 273, 565-571.

$\mathrm{H}$. Luders, SURFACTANT SCIENCE SERIES, 2000, 77-84.

F. Jérôme, S. Marinkovic and B. Estrine, ChemSusChem, 2018, 11, 1395 1409.

W. von Rybinski and K. Hill, Angewandte Chemie International Edition $1998,37,1328-1345$

16. K. Igarashi, in Advances in Carbohydrate Chemistry and Biochemistry Elsevier, 1977, vol. 34, pp. 243-283.

K. Hill, W. Von Rybinski and G. Stoll, Alkyl polyglycosides, Wiley Online Library, 1997

S.-i. Shoda, Proceedings of the Japan Academy, Series B, 2017, 93, 125-145. R. M. Schmaltz, S. R. Hanson and C. H. Wong, Chem Rev, 2011, 111, 42594307.

D. Svensson, S. Ulvenlund and P. Adlercreutz, Biotechnology and bioengineering, 2009, 104, 854-861.

M. S. U. Ackelid, presented in part at the Proc. Mat. Sci. Tech. Conf., Pittsburgh, PA, USA, 2009

F. Orata, Masinde Muliro University of Science and Technology.

P. van der Peet, C. T. Gannon, I. Walker, Z. Dinev, M. Angelin, S. Tam, J. E. Ralton, M. J. McConville and S. J. Williams, Chembiochem, 2006, 7, 13841391.

A. L. Morotti, K. L. Lang, I. Carvalho, E. P. Schenkel and L. S. C. Bernardes, Tetrahedron Letters, 2015, 56, 303-307.

25. K. Bock and C. Pedersen, Journal of the Chemical Society, Perkin Transactions 2, 1974, DOI: 10.1039/P29740000293, 293-297.

F. jérôme, K. Vigier, P. Amaniampong, J.-L. Clément, D. Gigmes, C. Mellet, J. García Fernández, Y. Blériot and G. Chatel, ChemSusChem, 2018, 11.

B. Estrine, S. Marinkovic and F. Jérome, 2019, DOI: 10.1016/b978-0-12 812705-6.00011-3, 365-385.

D. Geetha and R. Tyagi, Tenside surfactants detergents, 2012, 49, 417-427. W. R. K. Hill, Angew. Chem. Int. Ed., 1998, 37, 1328-1345.

S. K. Mamidyala and M. Finn, The Journal of organic chemistry, 2009, 74, $8417-8420$

31. V. K. Tiwari, B. B. Mishra, K. B. Mishra, N. Mishra, A. S. Singh and X. Chen Chem Rev, 2016, 116, 3086-3240.

J. Rosselgong, M. Chemin, C. C. Almada, G. Hemery, J. M. Guigner, G. Chollet, G. Labat, D. Da Silva Perez, F. Ham-Pichavant, E. Grau, S. Grelier, S. Lecommandoux and H. Cramail, Biomacromolecules, 2018, DOI: 10.1021/acs.biomac. 8 b01210. 
